\title{
Numerical Investigation of an UWB Localization Technique for Unmanned Aerial Vehicles in Outdoor Scenarios
}

\author{
Fabrizio Lazzari, Alice Buffi, Member, IEEE, Paolo Nepa, Member, IEEE, and Sandro Lazzari
}

\begin{abstract}
In this paper, the numerical investigation of an Ultra Wide Band (UWB) localization technique suitable for the tracking and control of an Unmanned Aerial Vehicle (UAV) in a specific outdoor scenario is presented. A set of UWB nodes are located on a moving/still ground station (GS) and interrogate an UWB node placed on the UAV that is flying in front of the GS. The distances between the GS-nodes and the UAV-node are estimated through a conventional two-way time-of-flight ranging method, one at a time, and then used in a multilateration algorithm. Due to the unavoidable relative motion between the UAV and the GS, above distances are actually measured for different UAV-GS relative positions, and then the UAV localization performance deteriorates as a function of the UAVGS relative speed and the ranging-method processing time. An approach is here proposed to mitigate above adverse effect, by exploiting an estimate of the UAV-GS relative speed along the GS forward direction. A preliminary numerical analysis is used to show that a decimeter order localization accuracy can be obtained for a tridimensional localization process.
\end{abstract}

Index Terms-Unmanned Aerial Vehicle, Ultra Wide Band localization, 3D localization, Two-Way Time-of-Flight, Time-of-Arrival algorithm.

\section{INTRODUCTION}

$\mathrm{U}$ NMANNED Aerial Vehicles (UAVs) [1] have received great attention thanks to their capability to reach places that are not easily accessible to humans. They can be employed in several application scenarios, such as military security, inventory control, search and rescue operations [2].

The correct navigation of such devices is a crucial aspect to manage the system and to preserve the human life in particular application scenarios. In this context, several systems can be employed, such as Inertial Navigation Systems (INS), Global Positioning Systems (GPS) or vision-based systems. In [3], the Extended Kalman Filter (EKF) is employed to determine the UAV location by exploiting data acquired from a GPS module. In [4], a new INS/GPS sensor fusion scheme based on state-dependent Riccati equation nonlinear filtering has been proposed. However, the GPS system is subject to jamming and interferences, thus the positioning may not be enough accurate for the specific application. In [5] and [6], a vision-based approach exploiting a particle filter and a three-nested Kalman filter have been proposed, respectively. The main advantage of a vision-based approach is that the system is passive and the UAV is typically already equipped with a camera. However, the weather conditions may affect localization performance.

As an effective alternative, the Ultra Wide Band (UWB) technology can be employed [7]. Indeed, it may guarantee a centimeter accuracy, by implementing a relatively low-power system [8]-[9]. Due to the high temporal resolution of UWB signals, time-based location estimation schemes usually provide better accuracy than those based on the angle of arrival [10] or the signal strength [8]. Among time-based methods, it is possible to distinguish the Time of Arrival (TOA) algorithm [11] and the Time Difference of Arrival (TDOA) algorithm. The TOA can be employed when the transmitting and the receiving nodes have a common clock, and the target node location is determined by intersecting a set of spheres. Instead, the TDOA only requires a synchronization between the reference nodes [12], and the target node position is determined by the intersection of hyperboloids. In [13], a performance comparison of TOA- and TDOA-based location algorithms in a Line-Of-Sight (LOS) environment is presented. To avoid synchronization among nodes, a Two-Way Time-of-Flight (TW-ToF) ranging method can be adopted [14]. In this case, the time of flight is measured at the transmitting node after receiving the backward signal from the receiving node, so avoiding the need for a synchronization. An UWB indoor-positioning system for UAV navigation exploiting the TW-ToF method has been proposed in [15]. In [16], a ground-based multi-sensor system (an UWB radar together with a laser pointer) has been proposed for the guidance and safe landing of an UAV in outdoor scenarios.

Manuscript received January 23, 2017, accepted March 5, 2017. This work was supported by Finmeccanica - Defence System Division.

Fabrizio Lazzari is with Italian Navy (e-mail: fabrizio.lazzari@marina.difesa.it).

Alice Buffi and Paolo Nepa are with the Department of Information Engineering, University of Pisa, Pisa 56122, Italy (e-mail: alice.buffi@iet.unipi.it, p.nepa@iet.unipi.it).

Sandro Lazzari is with Leonardo Company - Defence System Division (e-mail: sandro.lazzari@ leonardocompany.com). 
In this paper, a new UWB-based localization technique is presented, which is suitable for the tracking and control of an UAV in a particular outdoor scenario. Indeed, it is assumed that the UAV to be controlled is flying in front of a moving/still ground station (GS), which could be as the first vehicle of a military convoy. In particular, the automatic UAV control system on-board of the GS requires for the estimate of the instantaneous UAV relative position, to correctly fly the UAV along an assigned path or at a given distance from the moving GS. It is worth noting that, in such specific application, the UAV always flies in front of the GS, in LOS condition.

The paper is organized as follows: Section II presents a preliminary numerical analysis for choosing the best multilateration algorithm and the proper GS-node configuration. Then, in Section III, the new localization technique is described, together with a numerical investigation of the achievable localization performance. Finally, some conclusions are drawn in Section IV.

\section{PRELIMINARY ANALYSIS}

Let us consider an UAV flying in front of a GS, as for instance the first vehicle of a military convoy (Fig. 1). A set of UWB nodes are deployed onto the GS and they interrogate an UWB node on the UAV, one at a time, with an assigned order. The distance between each GS node and the UAV node is estimated through a TW-ToF ranging method, without requiring any synchronization among the UAV nodes. Then, by applying a multilateration algorithm, the tri-dimensional UAV position can be determined.

The geometry of the localization problem is shown in Fig. 1 for three different GS node configurations (the $z$-axis is parallel to the forward direction of the GS, while the $y$-axis denotes the altitude). In the following, four GS nodes have been considered, which is the minimum number of nodes required for a 3D localization. The origin of the coordinate system has been set in front of the GS.

The UAV location can be estimated by solving a set of nonlinear equations:

$\left[x_{S_{i}}-x_{U A V}\right]^{2}+\left[y_{S_{i}}-y_{U A V}\right]^{2}+\left[z_{S_{i}}-z_{U A V}\right]^{2}=r_{i}^{2}$

where $i=\{1,2,3,4\}, \underline{p}_{s_{i}}=\left[x_{s_{i}}, y_{s_{i}}, z_{s_{i}}\right]$ indicates the coordinates of each UWB node on the GS, $\underline{p}_{U A V}=\left[x_{U A V}, y_{U A V}, z_{U A V}\right]$ is the unknown UAV position, and $r_{i}=r_{i}+\varepsilon_{U W B}=\left|\underline{p}_{U A V}-\underline{p}_{S_{i}}\right|+\varepsilon_{U W B}$ is the measurement of the distance between the UWB node on the GS and that on the UAV. The latter is affected by the measurement error, $\varepsilon_{U W B}$, that is introduced by the TW-ToF ranging method implemented at the UWB node. Due to such an error, the solution of the nonlinear equations system (1) does not represent the intersection among four spheres at one point (ideal case), but it determines an intersection volume within which an approximate solution of (1) must be chosen. This phenomenon is represented in Fig. 2 for the bi-dimensional case with three nodes (trilateration problem). By considering the ideal case of exact distance measurements (dashed line in Fig. 2b) the three circumferences intersect in a single point coincident with the UAV position. On the contrary, in the real case of distance measurements corrupted by noise (solid line in Fig. 2b), the circumference intersection determines an area within which the UAV position has to be searched.

To properly characterize the error $\varepsilon_{U W B}$ used in the numerical analysis, a measurement campaign has been carried out by employing some commercial UWB PulsON 400 nodes by Time Domain [18], as illustrated in the Appendix. Based on the above characterization measurements, throughout the paper the error has been considered as a Gaussian aleatory variable with a null mean value and a variance $\sigma_{U W B}=1.4 \mathrm{~cm}: \varepsilon_{U W B} \in N\left(0, \sigma_{U W B}^{2}\right)$.

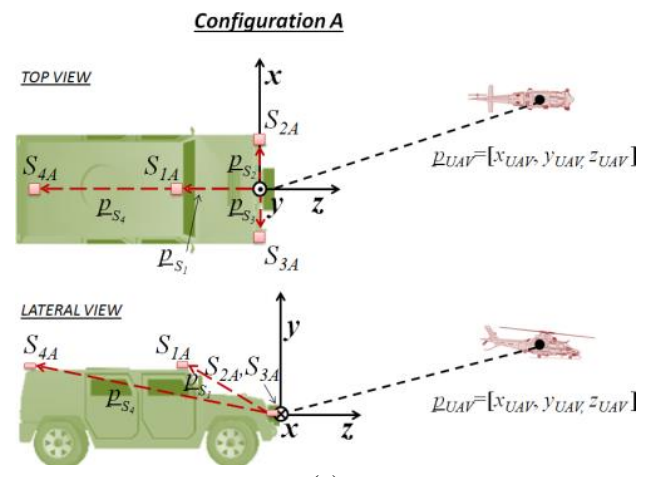

(a) 

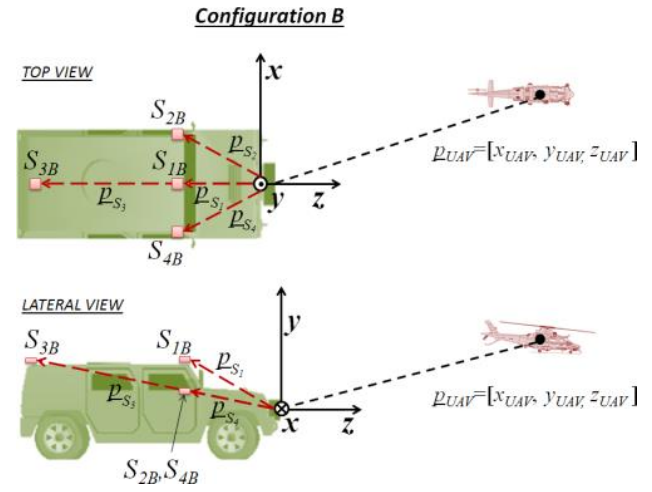

(b)
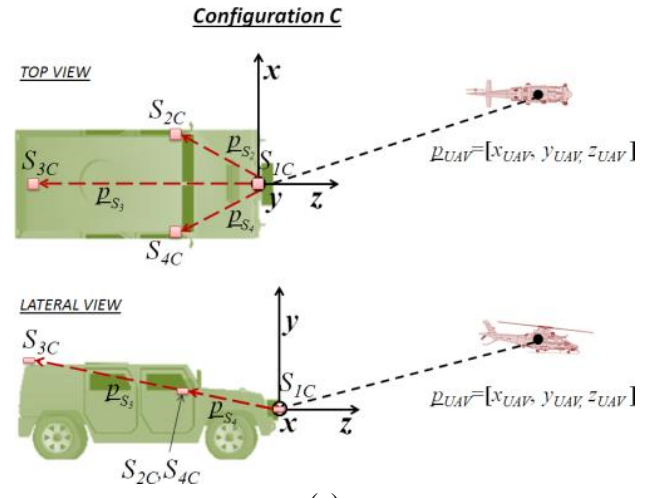

(c)

Fig. 1. Sketch of the ground station equipped with four UWB nodes and the UAV flying in front of it and equipped with one UWB node, for different configurations of the four UWB nodes onto the ground station.

Several methods exist to solve the system (1), and a comparison has been carried out by considering the Least Squares Method (LSM) [13], and the Levenberg-Marquardt algorithm (LMA) [19]-[20]. The latter is the combination of the gradient descendent algorithm and the Gauss-Newton algorithm. The numerical analysis has been carried out by employing the Matlab software. As preliminary tests the GS-node configuration A (Fig. 1a) has been employed with $S_{1 A}=[0,1,-1.2] m, S_{2 A}=[1,0,0] m$, $S_{3 A}=[-1,0,0] m$, and $S_{4 A}=[0,1,-4.8] m$. Results are shown in Fig. 3 in terms of the mean value of the absolute localization error for all three coordinates: $\varepsilon_{x}=\left|x_{U A V}-x_{U A V}\right|, \varepsilon_{y}=\left|y_{U A V}-y_{U A V}\right|$ and $\varepsilon_{z}=\left|z_{U A V}-z_{U A V}\right|$, where $\underline{p_{U A V}}=\left[x_{U A V}, y_{U A V}, z_{U A V}\right]$ is the estimated UAV position. The following UAV positions have been analyzed: $p_{U A V-1}=[0,1,5] m, p_{U A V-2}=[1,0.5,6] m$, $p_{U A V-3}=[0.5,1.5,10] m, p_{U A V-4}=[-0.5,2,12] m, p_{U A V-5}=[2,2.5,15] m$, and $p_{U A V-6}=[1.5,3,20] m$. For each UAV position, 300 Monte Carlo simulations have been performed and such number of test cases has been employed for each parameter configuration throughout the paper. It appears that the absolute error becomes larger when increasing the distance between the GS and the UAV, as expected (the sphere intersection volume becomes larger). Regarding the $x$-coordinate estimation, the two algorithms behave in the same way, but the LMA estimates the $y$ - and $z$-coordinate with a lower error (circle markers). When using the LSM (square markers), the mean error averaged among the considered UAV positions is $14 \mathrm{~cm}$, for all coordinates, while it decreases to $8 \mathrm{~cm}$ if the LMA is used. Therefore, the LMA has been employed throughout the paper to solve the multilateration problem (1). 

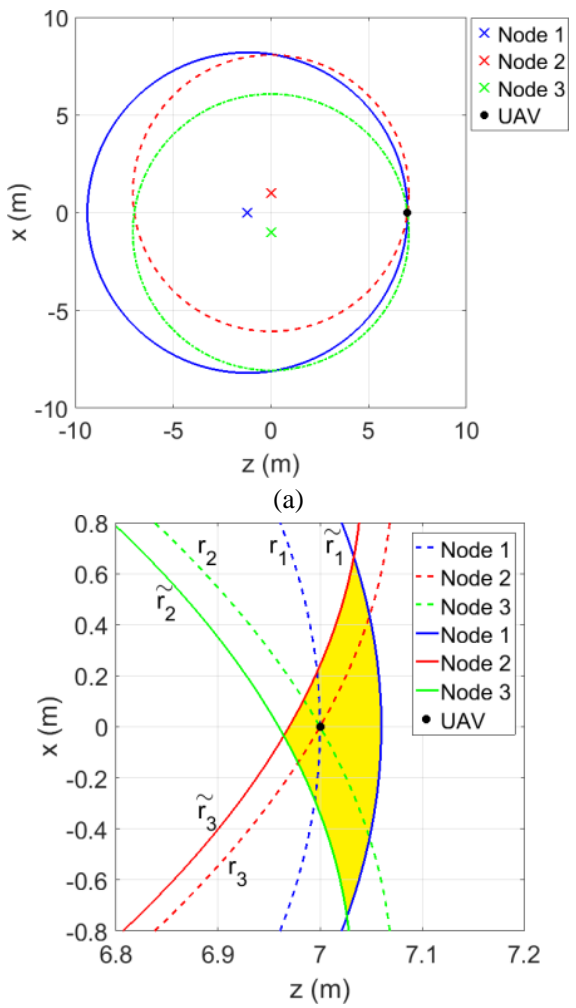

(b)

Fig. 2. (a) $2 \mathrm{D}$ representation of the trilateration problem related to the intersection among three circumferences. (b) Zoom around the intersection point among circumferences when distances are exactly measured (dashed line) and intersection area among circumferences when distances are corrupted by noise (solid line).

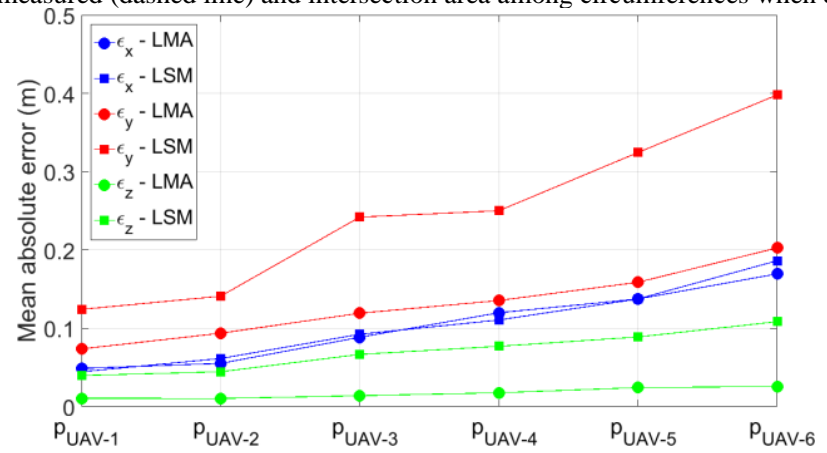

Fig. 3. Mean value of the UAV localization error for the three coordinates, by employing the LSM (square markers) and the LMA (circle markers) algorithms, for the following UAV positions: $p_{U A V-1}=[0,1,5] m, p_{U A V-2}=[1,0.5,6] m, p_{U A V-3}=[0.5,1.5,10] m, p_{U A V-4}=[-0.5,2,12] m, p_{U A V-5}=[2,2.5,15] m$, and $p_{U A V-6}=[1.5,3,20] m$.

\section{A. Analysis of the GS-node configuration}

As apparent from the results in Fig. 3, the error on the estimated UAV position can be of decimeter order even if it has been assumed an error of the measured distances of centimeter order (i.e. $\sigma_{U W B}=1.4 \mathrm{~cm}$ ). This is mainly due to the geometry of the particular scenario under analysis, where all the UWB reference nodes are grouped close to each other onto the GS, namely in a limited volume located at the back of the UAV (Fig. 2). This specific scenario is different with respect to a typical indoor scenario where all the reference nodes are spread out around the target node to be localized [21]. In Fig. 4, additional results are shown for the Configuration A' of the GS-nodes, which is similar to Configuration A except for the relative distance among the nodes: $S_{1 A}{ }^{\prime}=[0,3,-1.2] m, S_{2 A}{ }^{\prime}=[3,0,0] m, S_{3 A}{ }^{\prime}=[-3,0,0] m$, and $S_{4 A}{ }^{\prime}=[0,3,-7] m$. The mean value of the absolute localization error decreases for all three coordinates. As expected, localization performance may be improved by increasing the distance between the UWB nodes on the GS, once the restrictions imposed by the vehicle size and shape are accounted for.

The positioning performance also depends on the particular deployment of the nodes onto the GS. The GS-node Configuration A (Fig. 1a) has been compared with two other configurations:

- Configuration B, with $S_{1 B}=[0,1,-1.2] m, S_{2 B}=[1,0.5,-1.2] m, S_{3 B}=[0,1,-4.8] m$, and $S_{4 B}=[-1,0.5,-1.2] m$ (Fig. 1b); 
- Configuration C, with $S_{1 C}=[0,0,0] m, S_{2 C}=[1,0.5,-1.2] m, S_{3 C}=[0,1,-4.8] m$, and $S_{4 C}=[-1,0.5,-1.2] m$ (Fig. 1c).

The localization performance is shown in Fig. 5, when the set of UAV positions as in Fig. 3 have been considered. The zcoordinate absolute error is not shown, as it is less than $2.2 \mathrm{~cm}, 4.5 \mathrm{~cm}$ and $3 \mathrm{~cm}$ for configurations A, B and C, respectively. All configurations guarantee an accurate $x$-coordinate estimation, while Configuration A performs notably better for the $y$-coordinate. Therefore, only Configuration A has been selected throughout the following analysis.

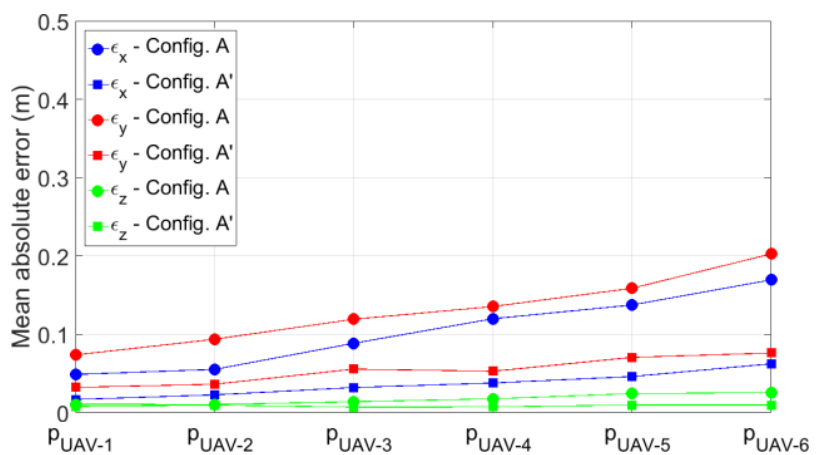

Fig. 4. Mean value of the UAV localization error for the three coordinates by employing the node Configuration A shown in Fig. 1a (circle markers) and the node Configuration A' (square markers), for the UAV positions as in Fig. 3.

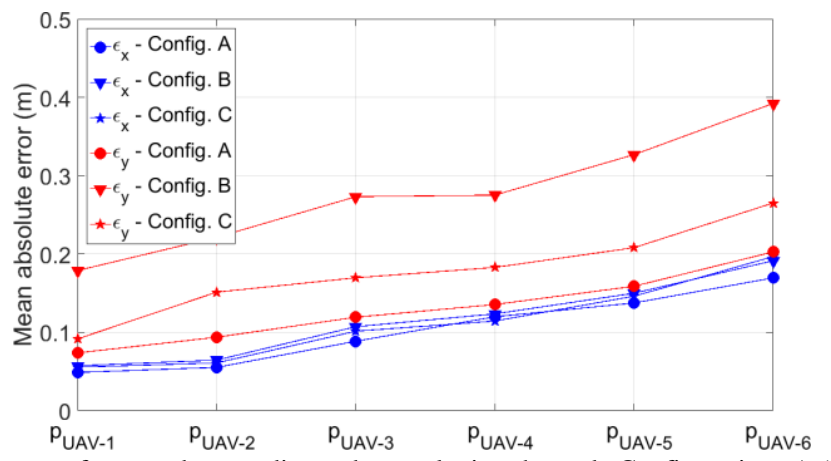

Fig. 5. Mean value of the UAV localization error for $x$ - and $y$-coordinates by employing the node Configurations A (circle markers), B (triangle markers) and C (star markers), for the UAV positions as in Fig. 3.

\section{LOCALIZATION ALgORITHM PERFORMANCE}

Generally speaking, the relative movements of the target node with respect to the reference nodes worsen the localization performance [17]. In the particular scenario here considered, a relative motion between the UAV and the GS usually exists. Then, during the time interval $\Delta t_{U W B}$ required by the UWB node to perform a single range measurement, the UAV moves and each distance is actually estimated for different UAV positions. A sketch of the problem is illustrated in Fig. 6.

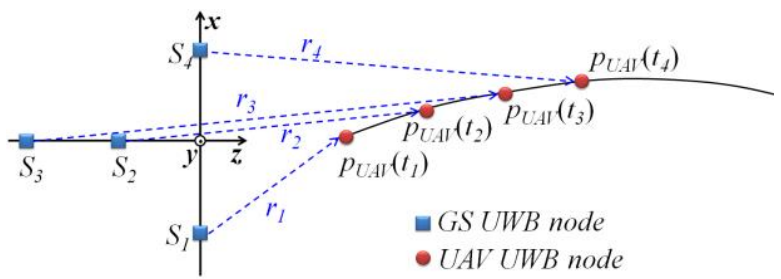

Fig. 6. Sketch of the distance vectors associated to the UWB nodes on the GS and the UWB node on the UAV, when a relative motion between the UAV and the GS does exist.

The first distance measurement is performed when the UAV is at position $\underline{p}_{U A V}\left(t_{1}\right)$, the second one is got after the UAV moved in a time interval $\Delta t_{U W B}$ to reach the position $\underline{p}_{U A V}\left(t_{2}\right)$, and so on. As a consequence, the relative distance between the UWB node on the GS and that on the UAV is also time dependent: $r_{i}\left(t_{i}\right)=\left|\underline{p}_{U A V}\left(t_{i}\right)-\underline{p}_{s_{i}}\right|$, and the solution of the problem in (1) corresponds to the intersection of spheres whose radius are actually measured with respect to different UAV positions.

To evaluate the effect of such phenomena, let us consider that the UAV is flying along a sinusoidal path in the $x z$-plane, as described in (2): 
$\left\{\begin{array}{c}x_{U A V}(t)=x_{U A V}(0)+\Delta x \cdot \sin (2 \pi \Delta v \cdot t / L) \\ y_{U A V}(t)=y_{U A V}(0) \\ z_{U A V}(t)=z_{U A V}(0)+\Delta v \cdot t\end{array}\right.$,

where $\left[x_{U A V}(0), y_{U A V}(0), z_{U A V}(0)\right]$ is the UAV relative position at the initial time, $\Delta x$ is the amplitude of the sinusoidal path, $L$ is the spatial period of the assumed sinusoidal path and $\Delta v$ is the UAV relative speed along the $z$-direction. Being the $z$-axis the advancing main direction, the speed components along the $x$ - and $y$-coordinates have been considered negligible. Simulation results that are obtained by solving the problem in (1) with the LMA approach, are shown in Fig. 7.

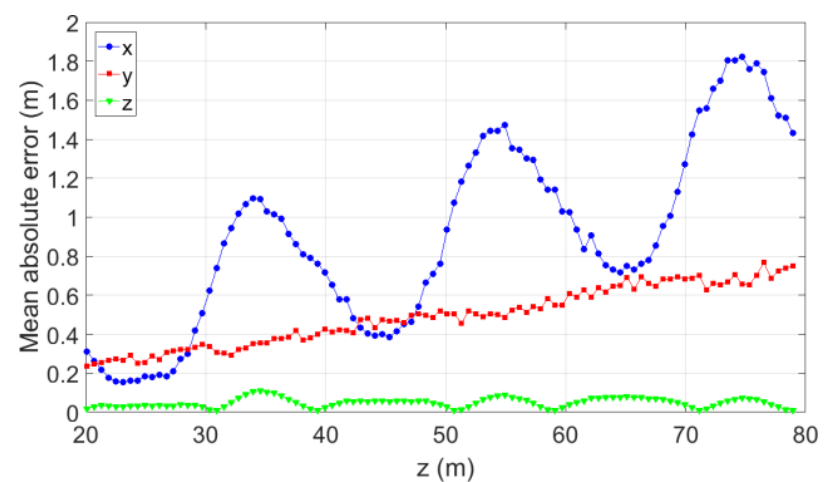

(a)

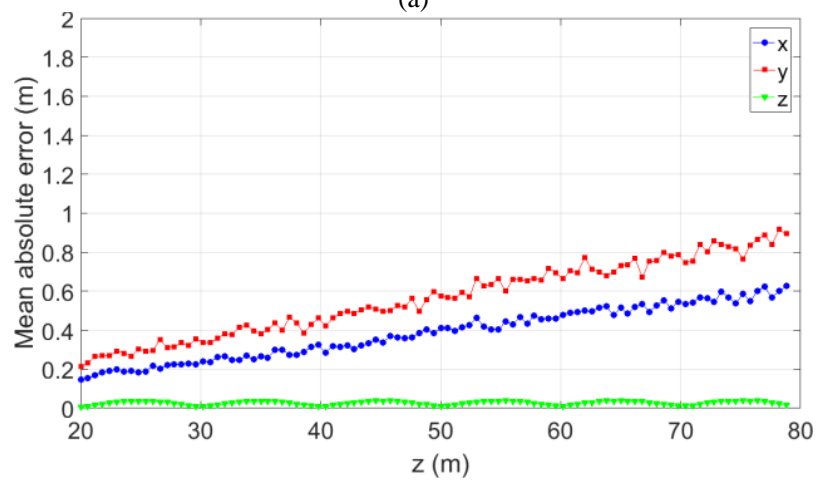

(b)

Fig. 7. Mean value of the UAV localization error for the three coordinates by considering: (a) the UAV motion during the distance measurements and $\Delta t_{U W B}=13 \mathrm{~ms}$; (b) the ideal case with simultaneous distance measurements $\Delta t_{U W B}=0$. An UAV sinusoidal motion in the $x z$-plane has been considered (see eq. (2)) and other system parameters are as follow: $\left[x_{U A V}(0), y_{U A V}(0), z_{U A V}(0)\right]=[0,1,20] \mathrm{m}, \Delta v=3 \mathrm{~m} / \mathrm{s}, \Delta x=5 \mathrm{~m}$ and $L=20 \mathrm{~m}$.

The following parameters have been used: $\Delta x=5 \mathrm{~m}, \Delta v=3 \mathrm{~m} / \mathrm{s}, L=20 \mathrm{~m}$; the observation interval is of $60 \mathrm{~s}$, corresponding to a variation of the $z$-coordinate in the interval $z \in[20,80] \mathrm{m}$ for the UAV initial position $\left[x_{U A V}(0), y_{U A V}(0), z_{U A V}(0)\right]=[0,1,20] \mathrm{m}$. From the datasheet of the UWB PulsON 400 nodes by Time Domain [18], it results that a time interval of around $13 \mathrm{~ms}$ is required to estimate distances up to $88 \mathrm{~m}$. Thus a time delay of $\Delta t_{U W B}=13 \mathrm{~ms}$ among consecutive measurements has been considered for the numerical results in Fig. 7a. To quantify the effect of the UAV movement with respect to the GS during each temporal interval $\Delta t_{U W B}$, the results in Fig. $7 \mathrm{~b}$ are those for the ideal case with $\Delta t_{U W B}=0$, namely when it can be assumed that all the GS nodes get simultaneously the distance measurement. The results in Fig. 7 also account for the distance error $\varepsilon_{U W B} \in N\left(0, \sigma_{U W B}{ }^{2}\right)$ with $\sigma_{U W B}=1.4 \mathrm{~cm}$. The $x$ - and $y$-coordinates, which are transverse with respect to the GS motion direction, exhibit a larger error, which increases with the distance between GS and UAV. By taking into account the UAV relative motion during the distance measurements (Fig. 7a), the absolute localization error worsen, especially for the $x$-coordinate, with respect to the ideal case of simultaneous distance measurements $\left(\Delta t_{U W B}=0\right)$ in Fig. 7b. This suggests that the effect of the UAV relative motion must be compensated to get a reliable positioning performance.

\section{A. Localization Algorithm with UAV Motion Compensation}

Numerical results in Fig. 7 showed that the $z$-coordinate is estimated with the lowest error, even when $\Delta t_{U W B} \neq 0$. Then, it 
follows that consecutive $z$-coordinate estimations can be used to get a reliable approximation of the UAV relative speed with respect to the GS. Indeed being the $z$-axis the advancing main direction, the speed estimation along such a direction will represent a good approximation of the total UAV speed. Let us consider two consecutive estimated UAV positions: $p_{U A V}\left(t_{n}\right)$ and $p_{U A V}\left(t_{n+1}\right)$, where $t_{n+1}=t_{n}+4 \Delta t_{U W B}$, as four UWB nodes on the GS have been considered. The UAV relative speed along the $z-$ direction can be approximated as in (3):

$\Delta v=\frac{z_{U A V}\left(t_{n+1}\right)-z_{U A V}\left(t_{n}\right)}{t_{n+1}-t_{n}}$

By the reasonable assumption that the UWB speed vector does not significantly change over a time interval equal to $4 \Delta t_{U W B} \simeq 52 \mathrm{~ms}$, the measured distances during the UAV relative motion can be corrected by subtracting the distance that the UAV travels along the forward direction. Thus, the improved distance estimation for each $i$-th node can be referred to the time of the first distance measurement $t_{n}$, namely to the UAV position at the first interrogation (4):

$r_{i}^{\prime}\left(t_{n}\right)=r_{i}\left(t_{i}\right)-\Delta v \cdot(i-1) \Delta t_{U W B}$,

with $i=\{1,2,3,4\}$ the node index. It is worth noting that the $i$-index is related to the instant at which the UWB node gets distance measurement, while the $n$-index is associated to the instant of the UAV position estimation, namely $t_{n}=t_{i}-(i-1) \Delta t_{U W B}$. With reference to Fig. 6, the first distance measurement $r_{1}$ is considered as is; at the second distance measurement, $r_{2}$ is subtracted a distance equal to $\Delta v \cdot \Delta t_{U W B}$, and so on. The localization performance obtained by using the measured distances $r_{i}$ and the improved ones $r_{i}{ }^{\prime}$ is shown in Fig. 8 and Fig. 9, respectively.

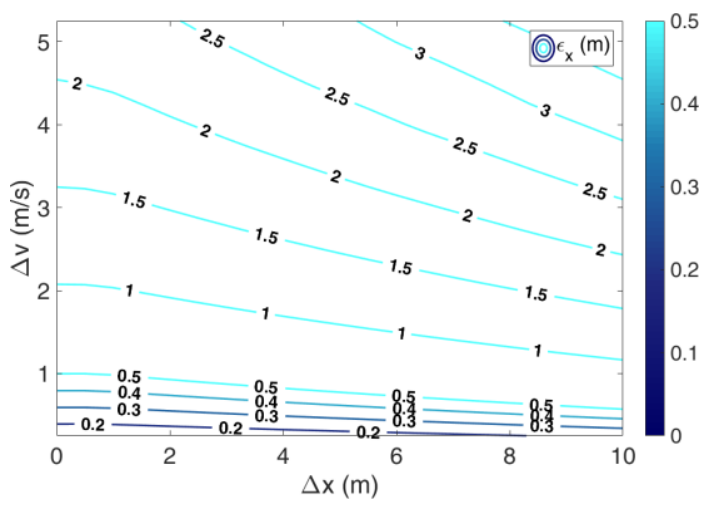

(a)

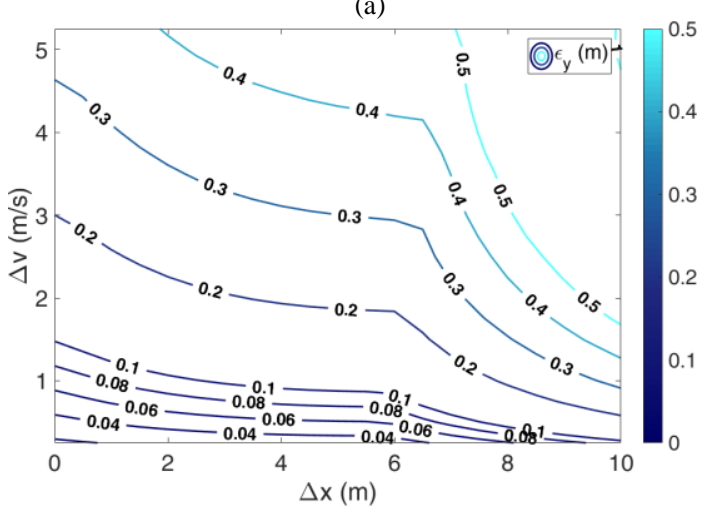

(b) 


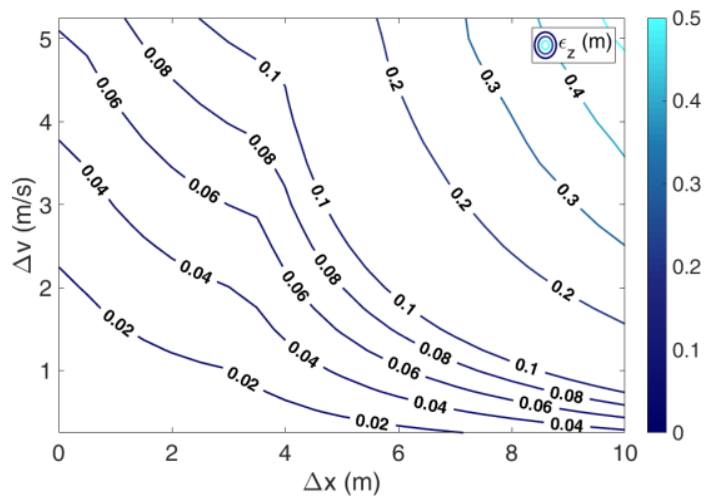

(c)

Fig. 8. Maximum localization error of the UAV, when it follows a sinusoidal trajectory in the $x z$-plane, by considering the UAV motion during the distance measurements, as a function of the sinusoid amplitude $\Delta x$ and relative speed $\Delta v:$ (a) $x$-coordinate, (b) $y$-coordinate and (c) $z$-coordinate. Node Configuration A has been considered and other system parameters are: $\left[x_{U A V}(0), y_{U A V}(0), z_{U A V}(0)\right]=[0,1,20] \mathrm{m}$ and $L=20 \mathrm{~m}$.
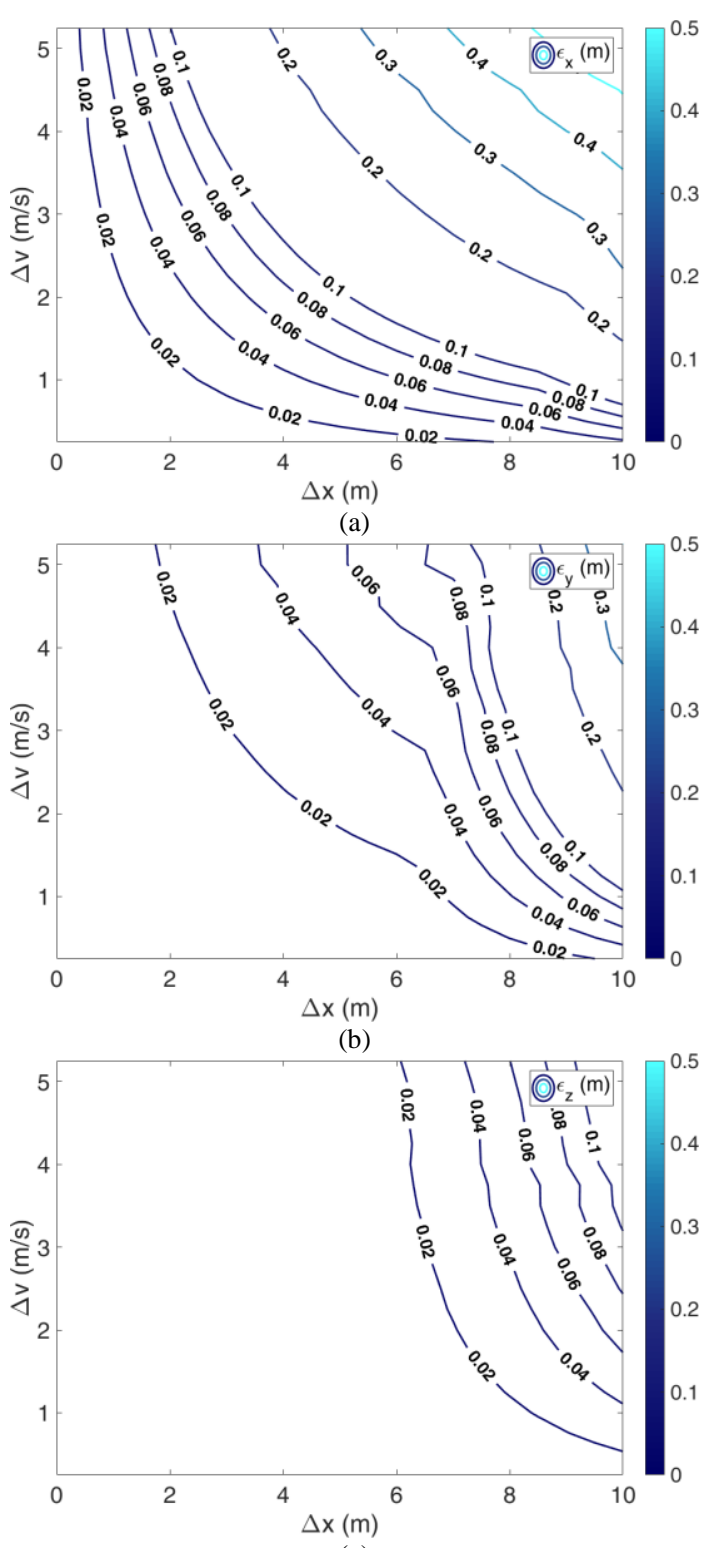

(c)

Fig. 9. Maximum localization error of the UAV, when it follows a sinusoidal trajectory on the $x z$-plane, by applying the proposed algorithm with the improved distances $r_{i}{ }^{\prime}$, as a function of the sinusoid amplitude $\Delta x$ and the relative speed $\Delta v:$ (a) $x$-coordinate, (b) $y$-coordinate and (c) $z$-coordinate. Node Configuration A has been considered and other system parameters are: $\left[x_{U A V}(0), y_{U A V}(0), z_{U A V}(0)\right]=[0,1,20] \mathrm{m}$, and $L=20 \mathrm{~m}$. 
Results are in terms of the maximum value of the UAV positioning error among all points of the sinusoidal trajectory in (2), when varying the amplitude of the assumed sinusoidal path in the interval $\Delta x=[0,10] \mathrm{m}$ and the UAV-GS relative speed in the interval $\Delta v=[0.25,5.25] \mathrm{m} / \mathrm{s}$. For $\Delta x=0 \mathrm{~m}$, the sinusoidal path (2) coincides with a uniform rectilinear motion of the UAV in front of the GS. Other system parameters are as follows: $L=20 \mathrm{~m}$ and $\left[x_{U A V}(0), y_{U A V}(0), z_{U A V}(0)\right]=[0,1,20] \mathrm{m}$.

If the UAV relative speed increases, the localization error worsen, especially for large $\Delta x$ values, and the $x$-coordinate is the more critical parameter. Without any compensation of the UAV motion, the localization error is less than $1 \mathrm{~m}$ and $0.5 \mathrm{~m}$, for the $y$-coordinate (Fig. 8b) and the $z$-coordinate (Fig. 8c), respectively, while for the $x$-coordinate the error can exceed $3.5 \mathrm{~m}$ (Fig. $8 \mathrm{a}$ ). Thanks to the employment of the new algorithm accounting for the UAV relative motion (4), it is possible to notably reduce the localization error for all the UAV coordinates. In particular, for the three considered cases, the maximum localization error is less than $50 \mathrm{~cm}$ and $30 \mathrm{~cm}$ for the $x$ - (Fig. 9a) and $y$-coordinates (Fig. 9b), respectively, while it is of centimeter order for the $z$ coordinate (Fig. 9c).

\section{B. Effect of the order of the GS-node interrogations}

The algorithm performance also depends on the specific order followed by the GS nodes when interrogating the UAV node. Fig. 10 shows the mean value of the localization error when the new algorithm is applied, for the $x$-and $y$-coordinates, for different GS-node interrogation orders. The UAV sinusoidal motion (2) has been considered with the following system parameters: $\Delta v=1 \mathrm{~m} / \mathrm{s}, \Delta x=5 \mathrm{~m}$ (Fig. 10a) and $\Delta v=2 \mathrm{~m} / \mathrm{s}, \Delta x=6 \mathrm{~m}$ (Fig. 10b).

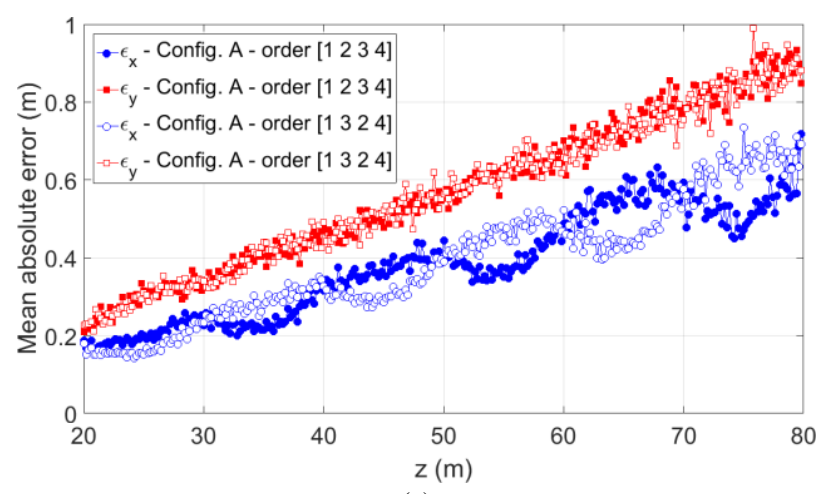

(a)

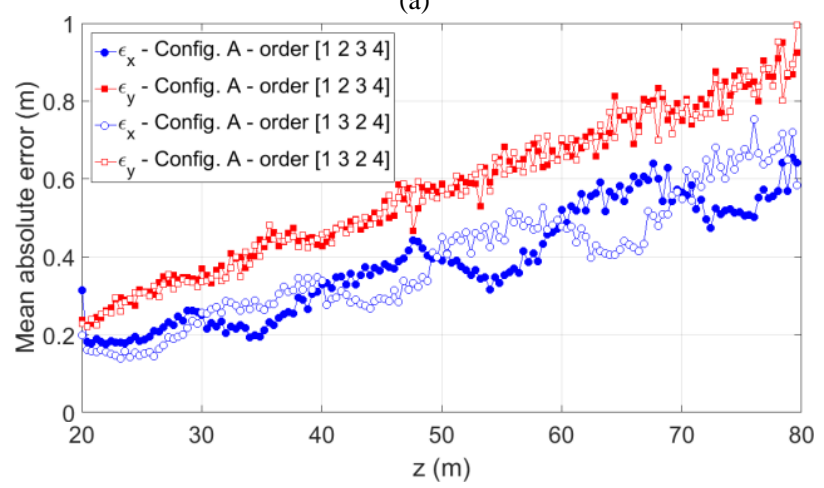

(b)

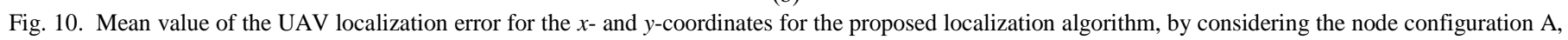

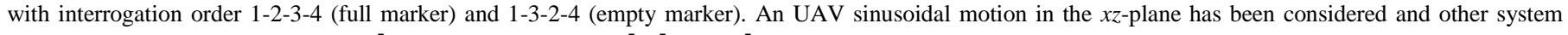
parameters are as follows: $L=20 \mathrm{~m},\left[x_{U A V}(0), y_{U A V}(0), z_{U A V}(0)\right]=[0,1,20] \mathrm{m}:$ (a) $\Delta v=1 \mathrm{~m} / \mathrm{s}, \Delta x=5 \mathrm{~m}$, and $(\mathrm{b}) \Delta v=2 \mathrm{~m} / \mathrm{s}, \Delta x=6 \mathrm{~m}$.

For the sensor Configuration A, the order 1-2-3-4 employed up to here (full marker), is compared with the order 1-3-2-4 (empty marker). The $y$-coordinate appears more robust with respect to the interrogation order. On the contrary, for some distance values, a specific configuration of the nodes determines a reduced error on the $x$-coordinate. As an example, at around $z=35 \mathrm{~m}$, the order 1-2-3-4 guarantees a lower error with respect to the order 1-3-2-4 and vice versa for other points along the trajectory (e.g. $z=45 \mathrm{~m}$ ) .

These results suggest the possibility to change the order at which the UWB nodes on the GS interrogate the UAV node along the trajectory. In particular, during the positive half-wave of the UAV trajectory, the order 1-3-2-4 allows a lower error, while during the negative half-wave, the order 1-2-3-4 permits an error reduction. Generally, a decimeter order improvement in the localization performance can be obtained, along the considered trajectories. 


\section{CONClusion}

An UWB localization technique suitable for tracking and control of UAVs in a specific outdoor scenario has been presented. Four UWB nodes placed on a GS interrogate a single UWB node on the UAV that flies in front of it, one at a time with an assigned order. Through a Two-Way Time-of-Flight ranging method (which does not require for a synchronization among the UWB nodes) the distance between each GS node and the UAV node is determined. Once above distance measurements have been improved by accounting for the GS-UAV relative speed, a multilateration algorithm is applied to estimate the 3D UAV position. A preliminary numerical analysis has shown that a decimeter order accuracy can be obtained for a tridimensional positioning of the UAV for relative distances up to $80 \mathrm{~m}$, when realistic values have been assumed for some parameters of the UWB nodes. The latter have been extracted from a measurement campaign with commercial nodes by Time Domain.

It is worth noting that the proposed technique does not require any synchronization among the UWB nodes, neither additional devices on the UAV for speed measurements (Inertial Measurement Unit).

Work is in progress to analyze the best node configuration on the GS, and the relationship between the interrogation order and the UAV path to reduce the localization error. Finally, the implementation of a tracking algorithm will be considered in a future work.

\section{APPENDIX}

To extract some realistic parameters to be used in the numerical analysis of the localization performance of the proposed algorithm, a measurement campaign has been carried out. Commercial UWB PulsON 400 nodes by Time Domain have been employed [18]. The measurement setup is shown in Fig. 11. Two nodes equipped with a linearly polarized antenna [22] have been placed facing each other in LOS condition. The transmitting sensor interrogates the receiving one, measures the two-way time-offlight, and then calculates the distance between the two nodes.

During the characterization, three node parameters have been varied: the Pulse Integration Index (PII), which is the power of 2 that determines the number of pulses that are transmitted for each symbol; the Transmit Gain (TG), which is a coefficient related to the transmit power; the distance $d$ between the two nodes. By considering $d=25 \mathrm{~m}, P I I=4$ and $T G=63,200$ measurements have been repeated and the cumulative distribution function (CDF) of the distance measurement error is shown in Fig. 12 (solid line).

Fig. 11. Measurement setup of two UWB PulsON 400 nodes by Time Domain.
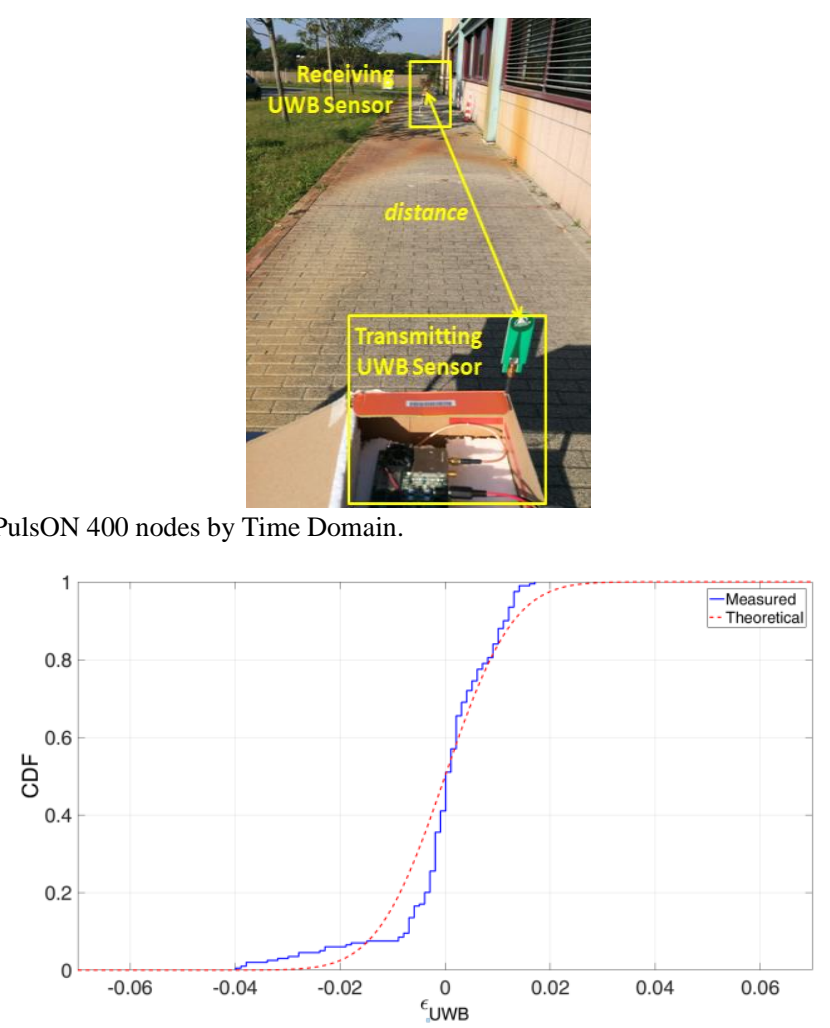

Fig. 12. Cumulative distribution function (CDF) of the distance measurement error obtained from 200 test cases with $d=25 \mathrm{~m}, P I I=4$ and $T G=63$ (solid line). The $\mathrm{CDF}$ of the Gaussian aleatory variable (dashed line) used in the numerical analysis of the localization performance of the proposed algorithm is also shown.

The UWB node ranging error, $\varepsilon_{U W B}$, has been modeled by a Gaussian aleatory variable with a null mean value and a variance $\sigma_{U W B}^{2}: \varepsilon_{U W B} \in N\left(0, \sigma_{U W B}^{2}\right)$. The CDF of the measured error has been compared with the CDF of a Gaussian aleatory variable (dashed line in Fig. 12) with mean value equal to zero and variance equal to that of the measured error. Repeated tests have been 
carried out by varying the system parameters in the following intervals: $P I I=\{4: 1: 10\}, T G=\{0,15,31,63\}$ and $d=\{5: 5: 60\}$ m. By considering 200 test cases for each configuration, a standard deviation of $\sigma_{U W B}=1.4 \mathrm{~cm}$ has been obtained.

It is worth noting that the PII value determines the maximum operating range of the UWB node as well as the time required to perform the range measurement. When changing $P I I$ from 4 to 6 , the operating range increases from $35 \mathrm{~m}$ up to $88 \mathrm{~m}$ but the processing time increases from $6.5 \mathrm{~ms}$ up to $12.5 \mathrm{~ms}$ [18]. Thus, depending on the application scenario a proper tradeoff value for the PII parameter has to be chosen.

\section{REFERENCES}

[1] V. Kumar and N. Michael, "Opportunities and challenges with autonomous micro aerial vehicles", The International Journal of Robotics Research, vol. 31, no. 11, pp. 1279-1291, 2012.

[2] S. J. Ingram, D. Harmer, and M. Quinlan, "UltraWideBand indoor positioning systems and their use in emergencies", 2004 Position Location and Navigation Symposium (PLANS 2004), pp. 706-715, 2004.

[3] S. C. Lee, W. R. Lee, and K. H. You, "TDOA based UAV Localization Using Dual-EKF Algorithm", Control and Automation-Communications in Computer and Information Science, vol. 65, pp. 47-54, 2009.

[4] A. Nemra and N. Aouf, "Robust INS/GPS Sensor Fusion for UAV Localization Using SDRE Nonlinear Filtering", IEEE Sensors Journal, vol. 10, no. 4, pp. 789-798, April 2010.

[5] J. Zhang, W. Liu, and Y. Wu, "Novel Technique for Vision-Based UAV Navigation", IEEE Transactions on Aerospace and Electronic Systems, vol. 47, no. 4, pp. 2731-2741, Oct. 2011.

[6] F. Kendoul, I. Fantoni, and K. Nonami, "Optic Flow-Based Vision System for Autonomous 3D Localization and control of small Aerial Vehicles", Robotics and Autonomous Systems, vol. 30, no. 6-7, pp. 591-602, June 2009.

[7] A. Benini, "Localization and Navigation of Autonomous Systems in Complex Scenarios", Doctoral Thesis, 2013 (http://openarchive.univpm.it/jspui/handle/123456789/1036).

[8] S. Gezici, Z. Tian, G. B. Giannakis, H. Kobayashi, A. F. Molisch, H. V. Poor, and Z. Sahinoglu, "Localization via ultra-wideband radios: a look at positioning aspects for future sensor networks", IEEE Signal Processing Magazine, vol. 22, no. 4, pp. 70-84, July 2005.

[9] S. J. Ingram, D. Harmer and M. Quinlan, "UltraWideBand indoor positioning systems and their use in emergencies", Position Location and Navigation Symposium (PLANS), 2004, pp. 706-715.

[10] B. G. Yu, G. Lee, H. G. Han, W. S. Ra, and T. W. Kim, "A Time-Based Angle-of-Arrival Sensor Using CMOS IR-UWB Transceivers", IEEE Sensors Journal, vol. 16, no. 14, pp. 5563-5571, July 2016.

[11] A. A. D'Amico, U. Mengali, and L. Taponecco, “TOA Estimation with the IEEE 802.15.4a Standard", IEEE Transactions on Wireless Communications, vol. 9, no. 7, pp. 2238-2247, July 2010.

[12] I. Guvenc and C. C. Chong, "A Survey on TOA Based Wireless Localization and NLOS Mitigation Techniques", IEEE Communications Surveys \& Tutorials, vol. 11, no. 3, pp. 107-124, 3rd Quarter 2009.

[13] G. Shen, R. Zetik, and R. S. Thoma, "Performance Comparison of TOA and TDOA Based Location Estimation Algorithms in LOS Environment", 5th Workshop on Positioning, Navigation and Communication (WPNC) 2008, Hannover, pp. 71-78, 2008.

[14] S. Lanzisera, D. Zats and K. S. J. Pister, "Radio Frequency Time-of-Flight Distance Measurement for Low-Cost Wireless Sensor Localization", IEEE Sensors Journal, vol. 11, no. 3, pp. 837-845, March 2011.

[15] J. Tiemann, F. Schweikowski and C. Wietfeld, "Design of an UWB indoor-positioning system for UAV navigation in GNSS-denied environments", 2015 International Conference on Indoor Positioning and Indoor Navigation (IPIN), pp. 1-7, Oct. 2015.

[16] W. Kong, D. Zhang, and J. Zhang, "A ground-based multi-sensor system for autonomous landing of a fixed wing UAV”, 2015 IEEE International Conference on Robotics and Biomimetics (ROBIO), Zhuhai, China, pp. 1303-1310, 2015.

[17] L. Hu and D. Evans, "Localization for Mobile Sensor Networks", Tenth Annual International Conference on Mobile Computing and Networking (MobiCom 2004), Philadelphia, September 26 - October 1, 2004.

[18] http://www.timedomain.com/P400_RCM_ProductBrochure_reduced.pdf

[19] K. Levenberg, "A Method for the Solution of Certain Non-Linear Problems in Least Squares", Quarterly of Applied Mathematics, vol. 2, pp. 164-168, 1944.

[20] D. Marquardt, "An Algorithm for Least-Squares Estimation of Nonlinear Parameters", SIAM Journal on Applied Mathematics, vol. 11, no. 2, pp. 431-441, 1963.

[21] B. Gulmezoglu, M. B. Guldogan, and S. Gezici, "Multiperson Tracking With a Network of Ultrawideband Radar Sensors Based on Gaussian Mixture PHD Filters", IEEE Sensors Journal, vol. 15, no. 4, pp. 2227-2237, April 2015.

[22] http://www.timedomain.com/datasheets/TD Broadspec Antenna.pdf 